\title{
Developing Standard Reference Materials for Analytical Electron Microscopy
}

\author{
Eric B. Steel*, Jeff Davis*, and Andrew Herzing*
}

* National Institute of Standards and Technology, 100 Bureau Drive, MS 8371, Gaithersburg, 20899-8371

The analytical electron microscope provides elemental analysis on a spatial scale of nanometers or better through the measurement of characteristic $\mathrm{x}$ rays and electron energy loss spectrometry. Quantitation can be performed by several methods, the simplest, if the film is thin enough, is through application of relative sensitivity factors, since (to a first approximation) the atomic number, absorption, and fluorescence effects, familiar in the analysis of bulk targets with the electron probe microanalyzer, can be neglected [1]. More complex approaches using first principles or absolute approaches [2] require more knowledge of the physics of the process or calibrating materials.

NIST first developed a Standard Reference Materials (SRM) 2063 [3] for this purpose in 1987 (SRM 2063) and reissued a compositionally similar but thinner material in 1993 (SRM 2063a) [4]. This reference material sold out of stock in the last few years and is in the process of being reissued. Since thin film technologies have changed significantly in the last 20 years, we are testing several approaches to both manufacture and characterization. Also the purpose of the standard has evolved as the technique has matured, changing from a chemical reference material used to advance the nascent AEM method to more of a quality assurance material to characterize and assure proper performance of the instrument.

Characteristics of an ideal Analytical Electron Microscope (AEM) standard

An ideal standard for AEM would have the following characteristics:

1) The composition must be known and contain a range of elements of non-interfering $x$ ray peaks and energy loss edges. Characteristic x-rays and edges should span the energy region typically used for analysis: approximately $200 \mathrm{eV}$ to $20 \mathrm{kV}$ for $\mathrm{x}$-rays and 100 to $1500 \mathrm{eV}$ for EELS edges. The uncertainty on the composition values should be significantly smaller than needed for typical AEM chemical analysis.

2) The film thickness should be uniform and known and fall within the thin film criteria (small matrix corrections and reasonable low loss/Plasmon and multiple scattering artifacts).

3) The composition should be measurable by at least one, and preferably more, independent chemical-analysis reference methods.

4) The standard should be resistant to chemical and physical alteration in the laboratory ambient environment, under vacuum, and while being analyzed under the electron beam.

5) The method of producing the sample should allow sufficient quantities of identical samples to be fabricated to supply needs as well as to make the cost reasonable.

Thin film deposition

The original SRM was manufactured using argon ion sputter deposition from a $18 \mathrm{~cm}$ diameter bulk mineral glass target onto a planetary substrate holder capable of containing hundreds TEM grid supported carbon films and five large (47 $\mathrm{mm}$ diameter) filter substrates for bulk chemical testing. This deposition unit is no longer available so two other deposition techniques have been tested: 
Pulsed laser deposition (PLD) and RF sputter deposition shown in figures 1 and 2 respectively. Attaining uniform mass-thickness is a challenge as seen in the images. Chemical composition appears uniform during initial heterogeneity characterizations on the microXRF, EPMA, and AEM. The deposition approach is being changed to overcome these obstacles.

\section{References}

[1] D.B. Williams, C.B. Carter, Transmission Electron Microscopy, Springer, New York, 2009.

[2] M. Watanabe, D.B. Willams, J. Microscopy, Vol. 221, Pt 2, 2006, pp 89-109.

[3] E.B. Steel et al., Analytical Electron Microscopy, San Francisco Press, San Francisco,1981, pp $65-70$.

[4] Certificate of Analysis, https://www-s.nist.gov/srmors/view_cert.cfm?srm=2063a.

[5] The PLD was performed by Peter Schenk, NIST Ceramics Division and the RF sputter deposition was performed at the NIST Center for Nanoscale Science and Technology.

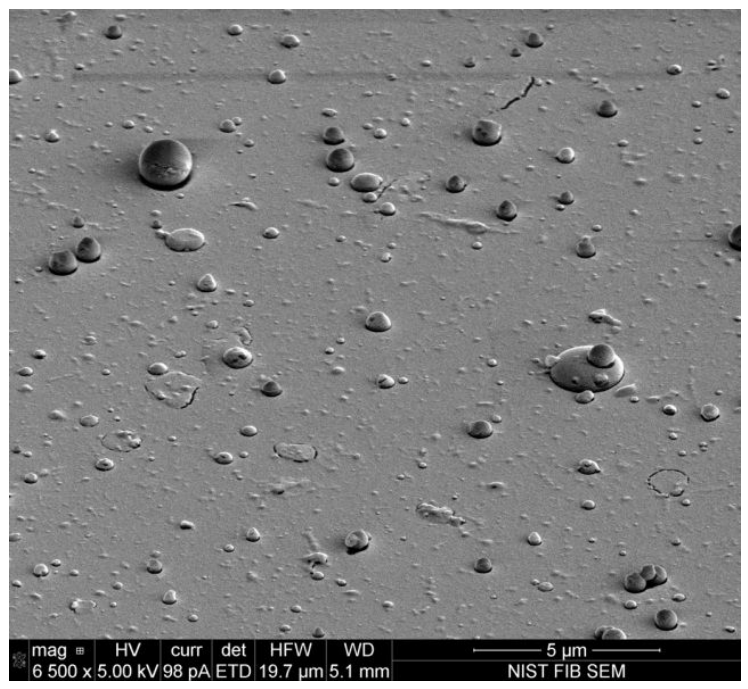

Figure 1 shows a secondary electron micrograph of a PLD film. Note the oblate spheroid and other particles deposited with the thin film. Cracks also appeared in this film deposited on a silicon wafer.

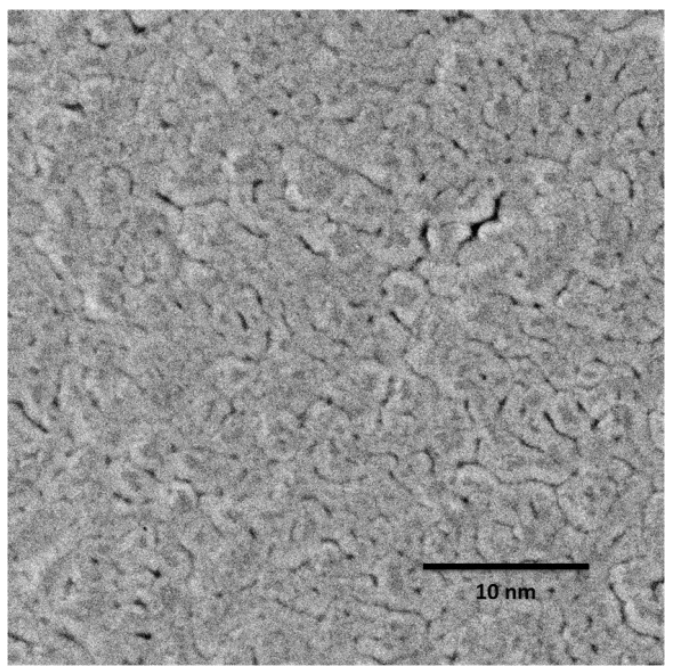

Figure 2 shows an AEM high angle darkfield detector scanning transmission micrograph of an RF sputtered film. 